\title{
Subject Index - HMORN 2008
}

Page numbers are followed by abstract numbers in parentheses.

Adherence, medication: 119 (C-A2-04), 125 (C-C1-05), 148 (PS2-23)

Administration: 119 (C-A1-01), 131 (PS1-01, PS1-02)

Adverse drug events: 140 (PS1-44)

Allergy: 142 (PS1-51)

Analytic methods: 123 (C-B3-01), 124 (C-B3-02, C-B3-03, C-B3-04), 125 (C-C1-06), 131 (PS1-07, PS1-08), 132 (PS1-09, PS1-10, PS1-11)

Anemia: 143 (PS2-05)

Antibiotics: 138 (PS1-36)

Arrhythmia: 144 (PS2-06)

Behavioral interventions: 133 (PS1-12, PS1-13, PS1-15, PS1-16)

Behavioral medicine: 124 (C-B3-03)

Bone mineral density testing: 127 (C-C3-01)

Cancer: 122 (C-B1-02), 123 (C-B1-03, C-B1-05), 132 (PS1-09), 134 (PS1-17, PS1-18, PS1-19), 135 (PS1-20, PS1-21, PS1-22, PS1-23), 136 (PS1-24, PS1-25)

Cancer screening: 136 (PS1-26)

Cardiometabolic risk factors: 121 (C-A3-03B)

Cardiovascular disease: 130 (C-D1-02, C-D1-03), 138 (PS1-31), 142 (PS1-54, PS2-01), 143 (PS2-03, PS2-04, PS2-05), 144 (PS2-06, PS2-08, PS2-09), 145 (PS2-11, PS2-12)

Carries risk: 139 (PS1-37, PS1-38)

Chronic diseases: 126 (C-C2-01)

Clinical: 113

Clinical practice: 140 (PS1-42)

Clinical trials: 135 (PS1-20)

Collaborative research: 151 (PS2-38)

Communication research: 132 (PS1-11)

Data collection: 121 (C-A3-02A), 123 (C-B1-03), 124 (C-B3-02)

Data management: 123 (C-B3-01)

Data simulation: 151 (PS2-37)

Dental health: 139 (PS1-37, PS1-38)

Depression: 126 (C-C2-05), 145 (PS2-13)

Diabetes: 120 (C-A2-09), 122 (C-A4-04), 124 (C-C1-02), 125 (C-C1-03, C-C1-04, C-C1-05, C-C1-06), 126 (C-C2-02), 127 (C-C3-03), 128 (C-D2-01), 133 (PS1-16), 140 (PS1-41), 142 (PS1-52), 145 (PS2-13, PS2-15), 146 (PS2-16, PS2-17)

Education: 119 (C-A2-02)

Elderly: 120 (C-A2-08), 144 (PS2-08)
Electronic health record: 128 (C-D2-03), 141 (PS1-50), 145 (PS2-11), 149 (PS2-29, PS2-30)

Electronic healthcare intervention: 130 (C-D1-02), 137 (PS1-27)

Evidence-based medicine: 124 (C-B3-04)

Health behavior: 121 (C-A4-03), 127 (C-C3-02)

Health disparities: 121 (C-A3-02A, C-A3-03B), 137 (PS1-27, PS1-28, PS1-29), 144 (PS2-09)

Health economics: 129 (C-C4-01, C-C4-04), 146 (PS2-18), 147 (PS2-19, PS2-20, PS2-21), 148 (PS2-22, PS2-23, PS2-24)

Health informatics: 127 (C-C3-01, C-C3-02, C-C3-03), 128 (C-C3-04, C-C3-05, C-D2-01, C-D2-03), 129 (C-D2-05), 131 (PS1-02, PS1-07, PS1-08), 148 (PS2-28), 149 (PS2-29, PS2-30, PS2-31), 150 (PS2-32, PS2-33, PS2-34), 151 (PS2-37, PS2-38, PS2-41)

Health insurance: 147 (PS2-19), 148 (PS2-24)

Health literacy: 123 (C-B1-05), 143 (PS2-03)

Health outcomes: 125 (C-C1-03)

Health promotion: 137 (PS1-30)

Healthcare: 113

Herpes zoster: 145 (PS2-15)

HIV: 129 (C-C4-01)

Hypertension: 133 (PS1-15)

Immunization: 137 (PS1-29)

Incidence rates: 121 (C-A4-01)

Integrated delivery of healthcare: 109

IRB: 130 (C-D4-01)

Managed care: 140 (PS1-45)

Managed care programs: 109

Management: 124 (C-C1-02), 125 (C-C1-04), 145 (PS2-12), 146 (PS2-16)

Medical library: 131 (PS1-01)

Medicare: 129 (C-C4-04), 134 (PS1-19), 146 (PS2-18), 147 (PS2-20, PS2-21)

Mortality risk: 130 (C-D1-03)

Multicenter study: 109

Multi-institutional research: 119 (C-A1-01), 130 (C-D4-01), 149 (PS2-31), 151 (PS2-41)

Obesity: 121 (C-A4-01, C-A4-03), 122 (C-A4-04, C-A4-05), 133 (PS1-13), 137 (PS1-30), 138 (PS1-31)

Orthopaedics: 141 (PS1-48)

Pharmaceutical prescribing: 141 (PS1-49) 
Pharmacoepidemiology: 119 (C-A2-02, C-A2-04),

120 (C-A2-05, C-A2-08, C-A2-09)

Physician education: 128 (C-C3-04)

Prescribing behavior: 138 (PS1-32, PS1-36)

Prevention: 143 (PS2-04)

Preventive medicine: 136 (PS1-26)

Privacy: 129 (C-D2-05)

Psychosocial adjustment: 134 (PS1-17)

Public health: 128 (C-C3-05)

Quality improvement: 126 (C-C2-01, C-C2-02, C-C2-05), 138 (PS1-32), 139 (PS1-39), 140 (PS1-41, PS1-42, PS1-44, PS1-45), 141 (PS1-48, PS1-49, PS1-50), 142 (PS1-51, PS1-52), 146 (PS2-17), 150 (PS2-32, PS2-34)

Quality of care: 150 (PS2-33)

Quality of life: 122 (C-B1-02), 135 (PS1-23), 136 (PS1-24)

Recruitment: 132 (PS1-10)

Recurrence risk: 136 (PS1-25)

Research: 113

Risk assessment: 120 (C-A2-05), 142 (PS2-01), 148 (PS2-28)

Risk factors: 122 (C-A4-05)

Smoking cessation: 137 (PS1-28), 148 (PS2-22)

Translational research: 134 (PS1-18), 139 (PS1-39)

Treatment: 135 (PS1-21, PS1-22)

Weight loss: 133 (PS1-12)

Youth: 142 (PS1-54) 